\title{
Phofonique silicium pour les télécoms et les réseaux
}

\author{
Philippe ABSIL \\ Directeur du Département Technologies Optiques \& 3D \\ IMEC, Louvain, Belgique \\ Ulrich MENGELE, ulrich.mengele@mengele-pmc.de
}

Le marché des dispositifs photoniques silicium a le vent en poupe. Une plateforme d'intégration photonique silicium, qui permet d'intégrer différents composants passifs et actifs de haute qualité en un seul flux intégré, permettra l'implémentation de circuits photoniques silicium. Grâce à cette plateforme, on peut produire des circuits intégrés photoniques offrant jusqu'à 25 Gbits/s par canal. Une bande passante globale très élevée peut être obtenue grâce à une architecture WDM (wavelength division multiplexing, ou multiplexage multi-longueurs d'onde), ou SDM (spatial division multiplexing, ou multiplexage spatial).

\section{Les liaisons optiques vont devenir indispensables}

Compte tenu de la croissance du trafic de données, les infrastructures ICT (information and communication technologies, ou télématique) de demain nécessiteront des bandes passantes encore plus élevées, et des communications offrant un meilleur rendement énergétique. La clé est la transmission optique. Les solutions à fibre optique sont déjà bien établies dans les réseaux d'accès et les réseaux urbains longue distance, ainsi que dans les infrastructures de supercalculateurs. Mais même les CPU (central processing unit) hautes-performances et les mémoires RAM (random access memory) dynamiques seront interfacées avec des entrées/sorties nécessitant des débits très élevés, de l'ordre de $1 \mathrm{To} / \mathrm{s}$ voire plus, d'ici 2018. Des liaisons optiques doivent donc être envisagées pour les interconnexions entre modules, et sur la puce elle-même. Pour toutes ces applications, l'avantage qu'apporte la transmission optique de données est une bande passante agrégée supérieure, pour une consommation moindre par rapport aux lignes de transmission en cuivre d'aujourd'hui.

Parmi les différentes technologies mettant en jeu des composants optiques, la photonique silicium est particulièrement intéressante car c'est elle qui offre le plus de potentiel en termes de réduction d'encombrement, faible consommation, coût minime et évolutivité. Selon un rapport récent de la société d'études de marché Yole Developpement (http:// www.i-micronews.com/reports/SiliconPhotonics-2014-report/1/445/), le marché de ces dispositifs photoniques Si va considérablement se développer dans les années à venir. Ce marché devrait passer de 25 millions de dollars en 2013, à environ 50 millions de dollars en 2017, pour dépasser 700 millions de dollars en 2024. Ce marché comprendra notamment le calcul hautes-performances, les data centers (centres de traitement de données) et les télécoms, ainsi que d'autres applications, comme certains capteurs dans le domaine médical ou grand-public. La photonique SI permet par exemple de réaliser des dispositifs optiques extrêmement sensibles, pour superviser des

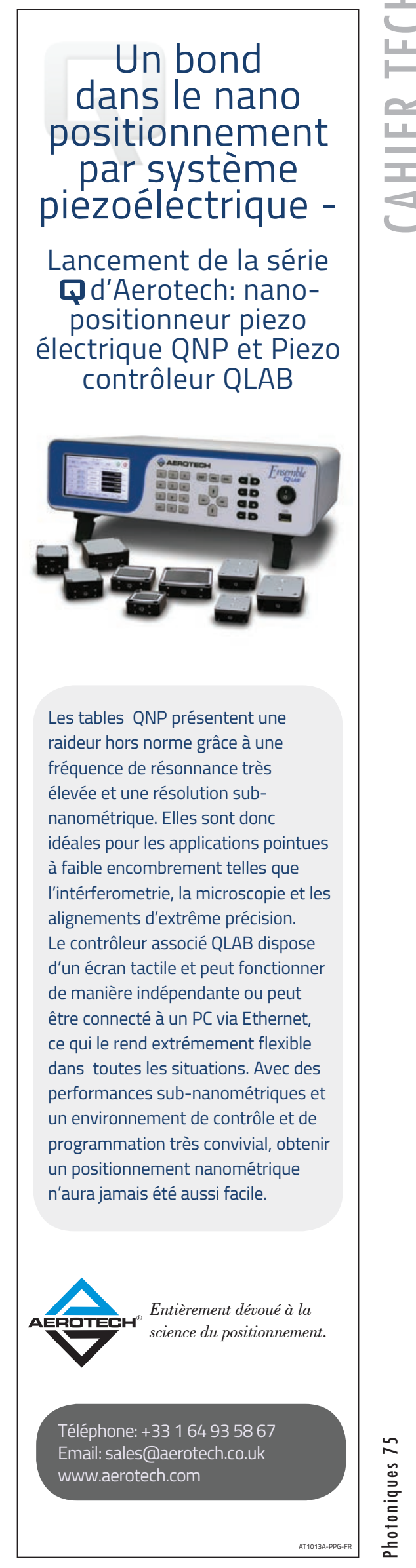




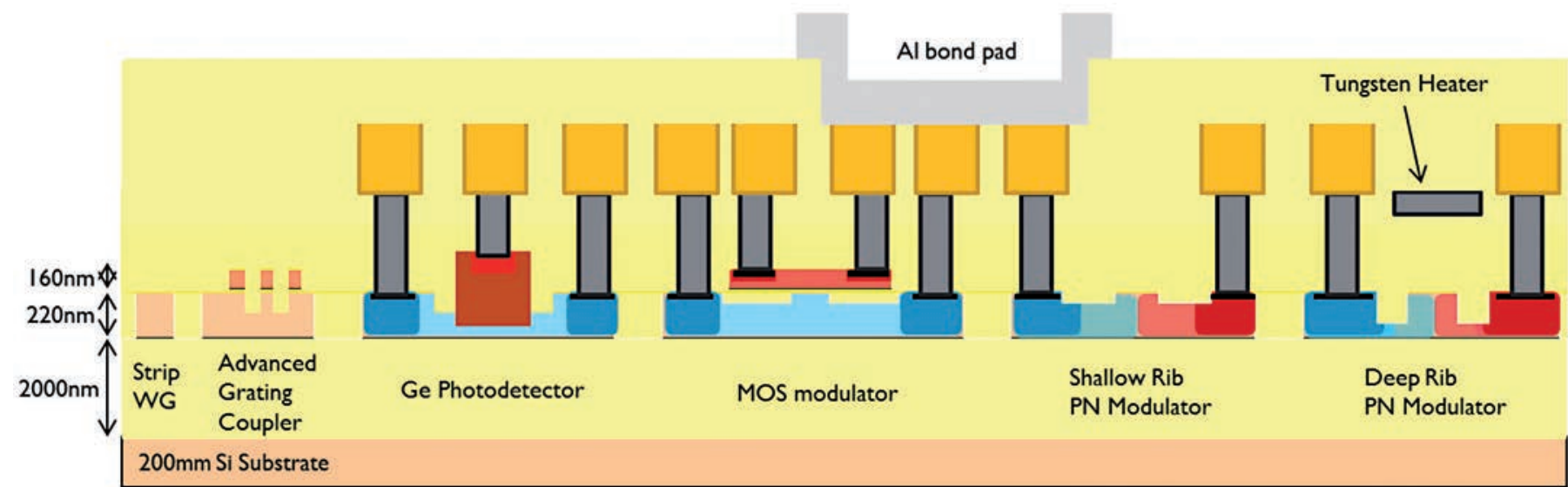

Figure 1. Schéma en coupe de la plateforme intégrée photonique silicium combinant des composants passifs de qualité, des modulateurs silicium, des réchauffeurs intégrés, et des photo-détecteurs Ge-on-Si.

contraintes mécaniques, des températures ou des gaz. Cette technologie peut également être utilisée dans des circuits très intégrés destinés à des dispositifs ou des implants médicaux.

\section{Technologie photonique silicium}

La plupart des réseaux de communication optiques utilisent la technique WDM, afin de minimiser le nombre de fibres nécessaires pour produire une bande passante donnée. Cette approche sert également aux liaisons optiques à faible distance, pour accroître la densité de bande passante entre les modules. Dans une liaison WDM, les signaux sont encodés sur plusieurs porteuses de longueurs d'onde différentes, qui sont ensuite transmises indépendamment sur le même canal physique. Les porteuses sont typiquement générées à l'aide de lasers de longueurs d'onde différentes. Un modulateur « imprime » ensuite le signal sur la porteuse, puis les signaux sont associés à l'aide d'un multiplexeur. Côté récepteur, les longueurs d'onde sont dé-multiplexées et envoyées chacune à un détecteur photoélectrique.

La photonique silicium à guide d'ondes est une technologie intéressante pour intégrer toutes les briques nécessaires pour construire des liens WDM sur une puce. Cette technologie permet de produire des composants optiques à l'aide d'équipements de pointe pour semiconducteurs, en utilisant les mêmes processus et les mêmes outils que pour la fabrication de puces. C'est aussi une technologie très difficile. En dehors du développement des différents composants passifs et actifs, l'intégration de ces composants en un circuit complexe, et au final intégré avec de l'électronique (drivers et amplificateurs), est loin d'être évidente. Les points faibles de la technologie photonique $\mathrm{Si}$ sont d'une part sa grande sensibilité aux dimensions du guide d'ondes (qui exige une maîtrise de réalisation au nanomètre près), et d'autre part la grande sensibilité de l'indice de réfraction Si aux variations de température (qui impose une régulation fine de la température, ce qui peut sérieusement impacter la consommation globale de la liaison optique).

\section{Plateforme d'intégration photonique silicium}

Les plateformes photoniques silicium les plus performantes permettent aujourd'hui la mise en place d'une architecture WDM complète. Elles utilisent des wafers en SOI de grand diamètre (i.e., $200 \mathrm{~mm}$ ) comme substrat, et une lithographie $193 \mathrm{~nm}$ pour toutes les couches de structuration de guides d'ondes. Une pile oxyde/poly-Si supplémentaire est déposée pour autoriser davantage de degrés de liberté dans la conception des composants optiques. Cette pile sert à intégrer les composants passifs complexes, comme les coupleurs et les guides

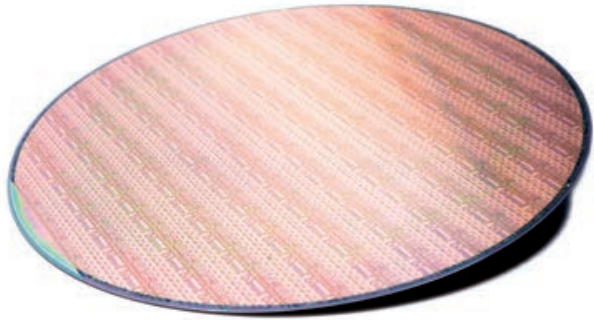

Figure 2. Circuits photoniques intégrés sur wafer, produits avec la plateforme d'intégration photonique silicium de l'IMEC.

d'ondes. Grâce à la plateforme d'intégration, des composants passifs ou des composants actifs (comme des modulateurs optoélectroniques, des réchauffeurs ou des photo-détecteurs Ge-on-Si) peuvent être co-intégrés. Les circuits électroniques (comme les drivers ou les TIA) peuvent être produits sur une puce séparée, puis montés sur les circuits photoniques $\mathrm{Si}$, en utilisant des techniques d'assemblage flip-chip (puce à retourner).

\section{Archilecture WDM à base de dispositifs microring}

L'architecture typique étudiée à l'IMEC contient une source laser à onde continue, couplée à un circuit photonique silicium à l'aide d'un coupleur à simple polarisation. La lumière WDM non-modulée peut être dédoublée à l'aide d'un répartiteur large bande pour « alimenter » deux liaisons parallèles. Chaque longueur d'onde est ensuite modulée par son propre 
résonateur microring, qui modifie son absorption, selon la tension du driver associé. Chaque modulateur peut fonctionner jusqu'à 25 Gbits/s. Ces modulateurs sont pilotés par des drivers CMOS alimentés en 1 volt. Ces modulateurs microring sont choisis pour leur compacité, qui limite la complexité et réduit la consommation. Un réchauffeur intégré sert à obtenir la longueur d'onde désirée. Le signal WDM est ensuite produit grâce à un unique coupleur de polarisation, et envoyé vers la matrice de fibres.

Le récepteur WDM est basé sur le démultiplexage du signal WDM à l'aide de doubles filtres microring du second ordre, à 4 canaux. L'utilisation de ce type de filtres permet d'atténuer l'impact de la variabilité du processus local sur les pertes d'insertion et sur les interférences. Tout d'abord, le signal est couplé au circuit photonique silicium par un coupleur répartiteur polarisant, qui envoie chaque polarisation vers un guide d'ondes différent. Ensuite, pour chaque polarisation, la longueur d'onde est filtrée et couplée à un photo-détecteur Ge-on-Si propre, suivie d'un TIA qui amplifie le courant photoélectrique généré par le détecteur. Les filtres sont réalisés à base de filtres microring, et sont accordés à la longueur d'onde désirée grâce aux réchauffeurs intégrés.

\section{Quelle est la prochaine étape?}

La technologie photonique Si s'avère être un candidat prometteur pour produire et intégrer les composants nécessaires à l'élaboration de liaisons optiques rapides pour les applications télécoms et réseaux. L'avenir appartient aux architectures WDM encore plus denses et encore plus efficaces au niveau énergétique. Pour cela, il faut améliorer encore l'isolation thermique des dispositifs critiques. En ligne de mire, un meilleur ajustement thermo-optique et une plage d'accordabilité spectrale plus étendue.

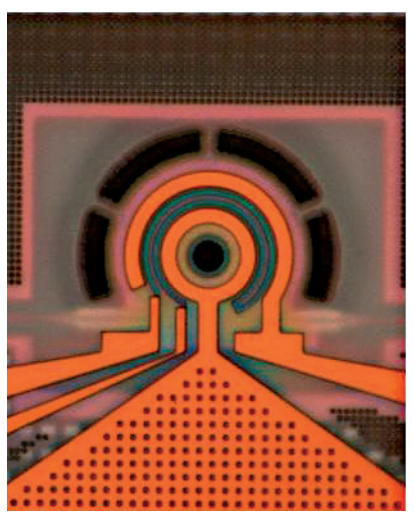

Figure 3. Image microscopique du modulateur microring obtenu à l'aide du module UCUT.

Pour en savoir plus
Advances in silicon photonics WDM devices,
Philippe P. Absil et al., Proc. SPIE 9010, Next-
Generation Optical Networks for Data Centers
and Short-Reach Links.
Highly uniform 28Gb/s Si photonics platform for
high-density, low-power WDM opt ical intercon-
nects, P. Verheyen et al., IPRSN Conference
paper, July 2014.

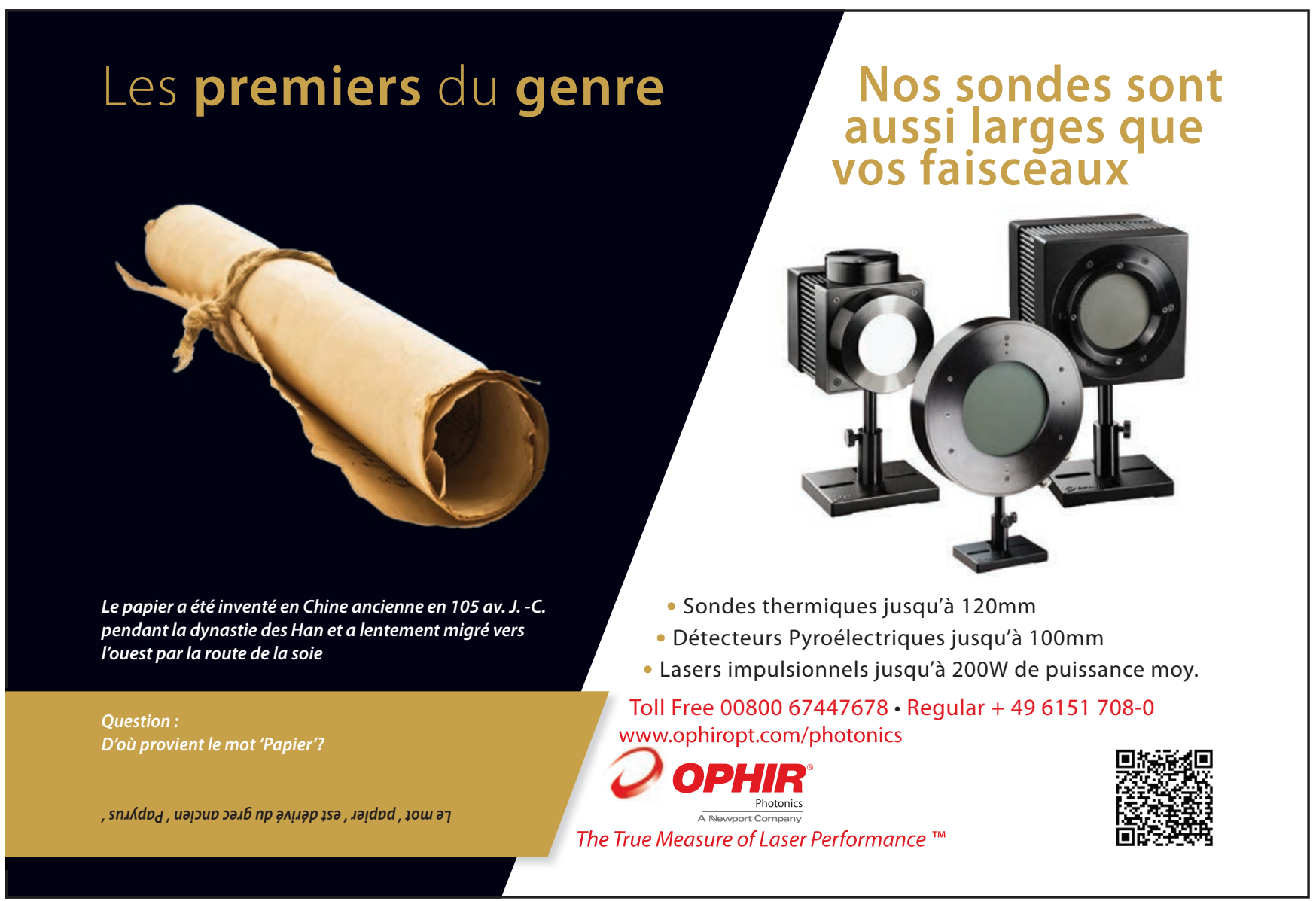

